\title{
Open adrenalectomy for medium sized adrenocortical tumour: How I do it?
}

\author{
Wael M. Sameh, MD; Ahmed Fouad Kotb, MD
}

Department of Urology, Alexandria University, Alexandria, Egypt

Cite as: Can Urol Assoc J 2015;9(5-6):E291-3. http://dx.doi.org/10.5489/cuaj.2572 Published online May 13, 2015.

\section{Abstract}

Introduction: The aim of our work was to report our experience in managing cases with medium-sized adrenocortical carcinoma by the high retroperitoneal extra pleural approach.

Methods: During the past 2 years, 10 patients with suspected adrenocortical carcinoma were managed by our technique: the high supra 10th rib, retroperitoneal extra pleural approach. We included cases with 5 to $10 \mathrm{~cm}$ adrenal masses, suspected as adrenocortical carcinoma.

Results: The mean patient age was 38 years (range: $26-44)$, the median tumour volume was $7 \mathrm{~cm}$ (range: 5-8). Of the 10 patients, 7 were female. Of the patients, 6 had right- and 4 had left-sided tumours. Intraoperatively, all cases had proper surgical removal, with no apparent residual tumour tissue. No single patient required a chest tube or developed respiratory problems. There were no major vascular injuries during surgery. We did not compare our findings to the standard lateral or subcostal approaches, as in our institution we adopt this high lateral approach for medium-sized tumours, while managing larger tumours with transperitoneal subcostal approach and smaller tumours laparoscopically.

Conclusion: The high supra 10th lateral retroperitoneal, extra pleural approach is a safe, doable technique, allowing easy access to medium-sized suprarenal tumours and its vasculature, for cases suspected to be adrenocortical carcinoma.

\section{Introduction}

Although laparoscopic adrenalectomy represents the standard of care in managing adrenal masses, open surgery still has a major role in management of adrenal masses, suspected to be adrenocortical carcinoma (ACC).

Allolio and colleagues ${ }^{1}$ published a clinical update on the management of ACC. They recommended open adrenalectomy for stages I to III to completely remove the adrenal mass; they also recommend the procedure be performed by an expert surgeon. Porpiglia and colleagues ${ }^{2}$ recently published a debate on the proper technique for managing ACC. They concluded that this debate will likely continue and that the most important issue is the ability to completely resect the tumour, leaving no residual mass - either by the open or laparoscopic technique.

Ramachandran and colleagues ${ }^{3}$ found that although laparoscopic surgery may be the standard of care in most institutions, urologists should be well-trained to do the proper surgical technique for suprarenal gland removal, due to the possible conversion from laparoscopy to open surgery. Recently, Miller and colleagues ${ }^{4}$ published their experience in managing ACC cases. They found that the rate of positive surgical margin was $50 \%$ and $18 \%$ for cases managed by laparoscopy and open surgery, respectively. They concluded that laparoscopy should not be attempted in cases suspicious or known to be ACC. Gaujoux and colleagues ${ }^{5}$ recently published a review on the role of laparoscopy in management of ACC. They showed that there is a high risk of tumour spillage during laparoscopic adrenalectomy, and so laparoscopy should be avoided in managing ACC.

Different approaches can be used for open adrenalectomy, including posterior, flank, anterior transperitoneal approaches, and thoracoabdominal approaches. Anterior incision offers good exposure, good access to vessels, but may be associated with prolonged ileus. Flank incision is a familiar incision to urologists, but the traditional incision over 12th or 11 th rib may not permit adequate exposure of the adrenal mass and its vasculature. The thoracoabdominal approach is associated with many complications that we have stopped using it.

The aim of our work was to report our experience in managing cases with medium-sized adrenocortical carcinoma by the high retroperitoneal extra pleural approach.

\section{Methods}

During the past 2 years, 10 patients with suspected adrenocortical carcinoma were managed by our technique - the 
high supra 10th rib, retroperitoneal extra pleural approach.

We included cases with a tumour size of 5 to $10 \mathrm{~cm}$. ACC was preoperatively suspected, using multiphasic computed tomographic adrenal protocol. To be included in the study, patients must have had an adrenal mass with an attenuation value of $>10 \mathrm{HU}$ on precontrast film, heterogeneous enhancement on contrast injection, and a washout at 15 minutes of $<50 \%$.

During surgery, the patient was put in the classic lateral position, with hyperextension of the flank. We did a supracostal incision, over the upper border of the 10th rib, with adequate dissection of the pleura and diaphragmatic fibres. No single case required resection of the rib. The peritoneum was kept intact during surgery. Figure 1 shows the exposure of the kidney, suprarenal tumour, with lung within uninjured pleura. On the left side, we identified the left renal vein, dissected it and accessed the left suprarenal vein that we ligated. On the right side, we performed an adequate dissection of the inferior vena cava (IVC), and then we did a lateral traction on the suprarenal gland, identified and ligated the right suprarenal vein. Figure 2 shows the ligated right suprarenal vein. We then completed the dissection of the gland until it was completely removed. Figure 3 shows the clean bed, following adrenalectomy.

\section{Results}

The mean patient age was 38 years (range: $26-44$ ), the median tumour volume was $7 \mathrm{~cm}$ (range: 5-8). Of the 10 patients, 7 were female. Of the patients, 6 had right- and 4 had left-sided tumours. The mean follow-up of patients was $13.6 \pm 6$ months (range: $6-22$ ).

Intraoperatively, all cases had proper surgical removal, with no apparent residual tumour tissue. Two patients had

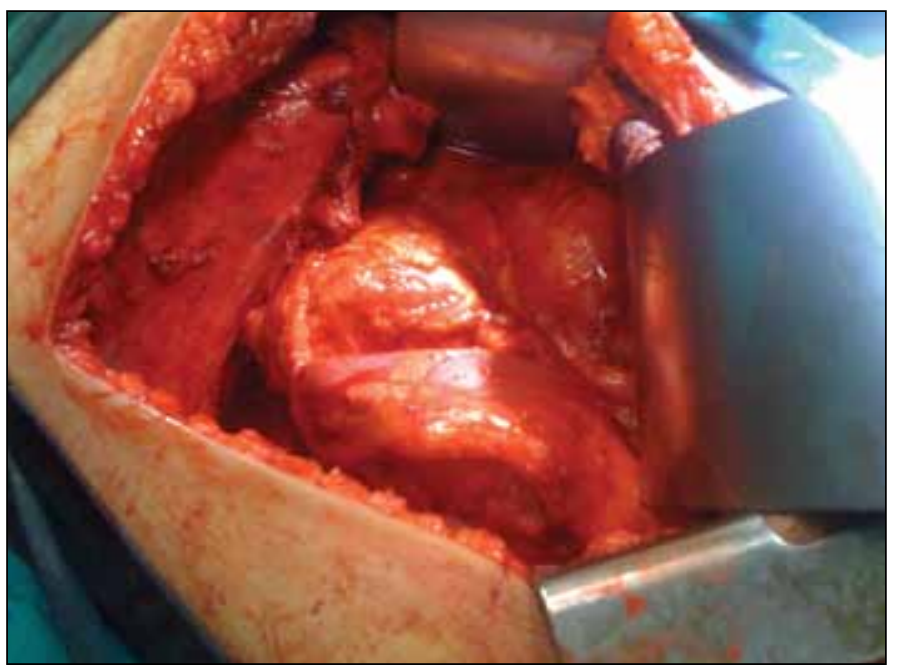

Fig. 1. Full exposure of the right suprarenal tumour with apparent lower part of the right lung within the uninjured pleura. their pleura injured during the incision and dissection of the pleura. In both patients, the pleural tear was identified immediately and closed immediately under positive airway pressure. No single patient required chest tube or developed respiratory problems. No major vascular injury happened during surgery. Complete tumour resection was achieved for all cases, with no tumour spillage. No single case required nephrectomy or splenectomy for the complete mass removal.

Retroperitoneal tube drain was left for 48 hours. The hospital stay was 1 day for all patients, during which nonsteroidal anti-inflammatory drugs were used at fixed times, with added narcotics (nalorphine) as needed. Oral diet was resumed on the night of the surgery. No postoperative surgical complications were encountered, except for pain related to the incision and managed by non-steroidal antiinflammatory drugs. Chest X-ray was done before discharge and confirmed no intra pleural collection. Pathologically, all patients had ACC.

At our institution, we follow such patients with a computed tomography every 6 months. All patients in our study were tumour free during follow-up, except one female who developed local recurrence and multiple pulmonary deposits, detected on the first radiological investigations after 6 months. This patient received mitotane and showed no response during follow-up, with progression of metastases. She is currently on regular pain killers. All patients, including the female with recurrence, are currently alive.

\section{Discussion}

At our referral centre, we are fortunate to deal with many cases of ACC. Generally, we prefer the anterior subcostal transperitoneal approach to manage such an aggressive

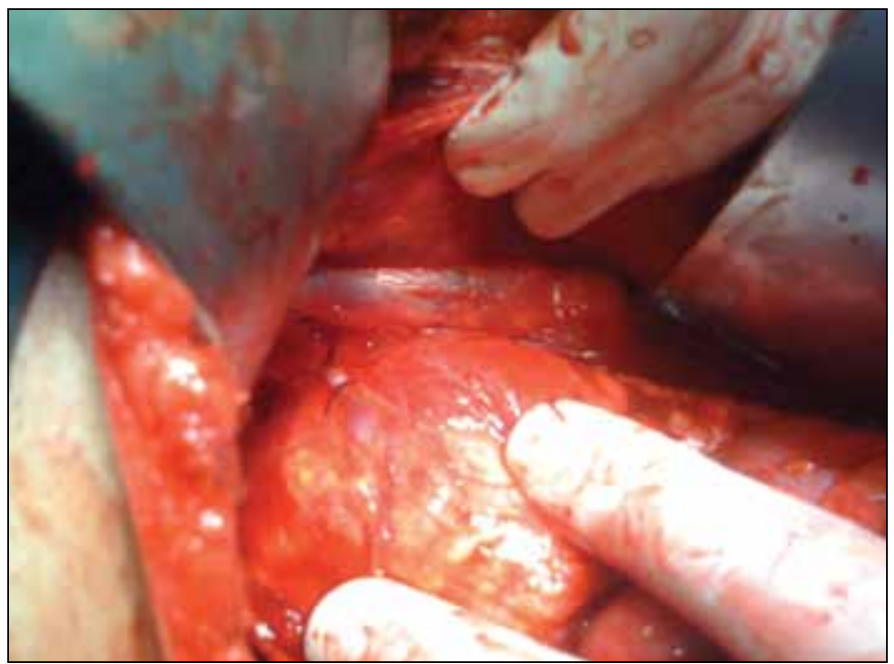

Fig. 2. Suprarenal mass, inferior vena cava, and ligated right suprarenal vein. 


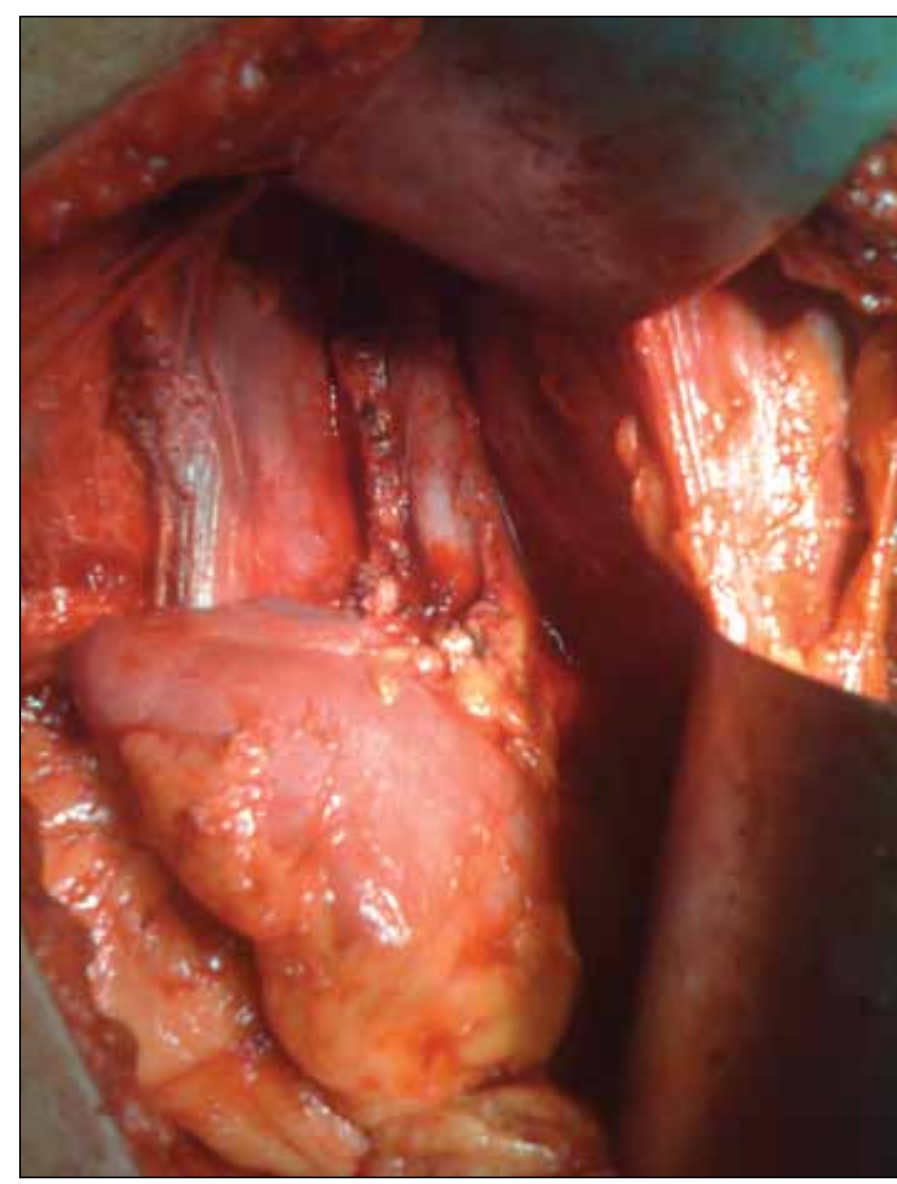

Fig. 3. Right kidney, inferior vena cava, and clean bed, following complete removal of the right suprarenal tumour.

tumour. Using this technique, we are able to mobilize the liver, get good exposure of the suprarenal mass regardless of its size, and easily access the great vessels for vascular control. The only actual problem we encounter, in a group of patients, is prolonged ileus. The lateral retroperitoneal approach above 12 th or 11 th rib, from our experience, is not suitable at all for open adrenalectomy. The adrenal tumour is usually high and has a medial extension, making its blind dissection an undesired option and may not allow real proper complete surgical removal. Another issue to consider is the risk of peritoneal spillage. Gonzalez and colleagues ${ }^{6}$ reported peritoneal spillage in $8 \%$ and $83 \%$ for cases managed by open and laparoscopic adrenalectomy, respectively. Leboulleux and colleagues ${ }^{7}$ reported 4 years postoperative peritoneal carcinomatosis of $26 \%$ and $66 \%$ of patients managed by open and laparoscopic adrenalectomy, respectively. At our institution, we do not offer laparoscopic adrenalectomy for cases suspected of ACC, except in selected cases of stage 1 disease, and these are performed by an experienced laparoscopic surgeon.

Due to the risk of prolonged ileus and the possible risk of peritoneal spillage, we decided to preserve the anterior subcostal approach for large tumours $>10 \mathrm{~cm}$, locally invasive tumours, and tumours with IVC thrombus. We could achieve complete tumour resection for all patients. We were not confronted with an accidental tumour rupture or spillage during the surgery. The postoperative period was smooth and oral fluids and soft diet were started safely for patients the night of the surgery.

In our cases, the supra 10th rib, retroperitoneal approach was a feasible technique, allowing easy access to the upper pole of the adrenal gland, and good access to draining vessels. In $80 \%$ of cases, it was possible to perform a retroperitoneal incision, without pleural injury, with careful dissection. Accidental pleural injury occurred in $20 \%$ of cases and it was identified and repaired immediately. No single patient developed pneumothorax or pleural effusion. There were no major vascular injury during the procedure and we were very comfortable with the vascular control achieved.

\section{Conclusion}

The high supra 10th lateral retroperitoneal, extra pleural approach is a safe, doable technique, allowing easy access to medium-sized suprarenal tumours and its vasculature, for cases suspected as ACC.

Competing interests: The authors declare no competing financial or personal interests.

This paper has been peer-reviewed.

\section{References}

1. Allolio B, Fassnacht M. Clinical review: Adrenocortical carcinoma: Clinical update. J Clin Endocrinol Metab 2006;91:2027-37. http://dx.doi.org/10.1210/ic.2005-2639

2. Porpiglia F, Miller BS, Manfredi M, et al. A debate on laparoscopic versus open adrenalectomy for adrenocortical carcinoma. Horm Cancer 2011;2:372-7. http://dx.doi.org/10.1007/s12672-011-0095-1

3. Ramachandran MS, Reid JA, Dolan SJ, et al. Laparoscopic adrenalectomy versus open adrenalectomy: Results from a retrospective comparative study. Ulster Med J 2006;75:126-8.

4. Miller BS, Ammori JB, Gauger PG, et al. Laparoscopic resection is inappropriate in patients with known or suspected adrenocortical carcinoma. World J Surg 2010;34:1380-5. http://dx.doi.org/10.1007/ s00268-010-0532-2

5. Gaujoux $S$, Bertherat J, Dousset B, et al. Laparoscopic adrenalectomy for adrenocortical carcinoma: A medico-surgical perspective. Ann Endocrinol (Paris) 2012;73:441-7. http://dx.doi.org/10.1016/i. ando.2012.07.001. Epub 2012 Sep 7.

6. Gonzalez RJ, Shapiro S, Sarlis N, et al. Laparoscopic resection of adrenal cortical carcinoma: A cautionary note. Surgery 2005;138:1078-85. http://dx.doi.org/10.1016/i.surg.2005.09.012

7. Leboulleux $S$, Deandreis D, Al Ghuzlan A, et al. Adrenocortical carcinoma: Is the surgical approach a risk factor of peritoneal carcinomatosis? Eur J Endocrinol 2010;162:1147-53. http://dx.doi.org/10.1530/ EJE-09-1096

Correspondence: Dr. Ahmed Fouad Kotb, Department of Urology, Alexandria University, Alexandria, Egypt; drahmedfali@gmail.com 